\title{
Sliding Mode Implementation of an Attitude Command Flight Control System for a Helicopter in Hover
}

\author{
D. J. McGeoch, E. W. McGookin, S. S. Houston
}

This paper presents an investigation into the design of a flight control system, using a decoupled non-linear sliding mode control structure, designed using a linearised, $9^{\text {th }}$ order representation of the dynamics of a PUMA helicopter in hover. The controllers are then tested upon a higher order, non-linear helicopter model, called RASCAL. This design approach is used for attitude command flight control implementation and the control performance is assessed in the terms of handling qualities through the Aeronautical Design Standards for Rotorcraft (ADS-33). In this context a linearised approximation of the helicopter system is used to design an SMC control scheme. These controllers have been found to yield a system that satisfies the Level 1 handling qualities set out by ADS-33.

Keywords: helicopter, sliding mode control, hover, handling qualities, ADS-33E-PRF, response types.

\section{Introduction}

The issue of helicopter flight control has been discussed extensively in the relevant literature [1, 2, and references therein]. Due to the complexity of helicopter dynamics, the design and implementation of controllers is difficult. Helicopters are highly coupled systems. The rotor provides propulsion and is the main control actuator, and is therefore the source of much of the complexity. The level of detail used to represent the rotor dynamics is often an important factor during the design of the controller and selection of the associated parameters $[3,4]$. As the flight conditions change, these dynamics change, often resulting in controllers that only perform to specification within the operation margin for which they are designed. All these factors have stimulated this area of research and resulted in a study of various control strategies being applied to this application. The goal is to achieve high-bandwidth, high-gain, robust controllers operable over the entire flight envelope [5].

One area of control that has had little application to the helicopter problem is that of non-linear, variable structure methods, such as Sliding Mode control, (SMC) [6, 7, 8, 9]. SMC is comprises of two parts: a linear equivalent term and a non-linear switching term. This non-linear term is the unique attribute for this type of control scheme. It provides much of the controllers' actuation power and provides high robustness to model uncertainty and external disturbance. However, it is also often the source of concern for this controller structure as the non-linear term tends to switch around the zero error region giving a high frequency input to the control actuator, called chattering $[10,11]$, which can be avoided by employing a soft switching structure.

The application of a SMC scheme to a helicopter system is presented in this paper. The controller is evaluated using the Aeronautical Design Standard performance specification of handling qualities requirements for military rotorcraft, ADS-33E [12].

\section{Helicopter model}

The RASCAL model (Rotorcraft Aerodynamics Simulation for Control Analysis) [13] is used to represent the helicopter dynamics in this study. This is a high order, nonlinear, individual rotor blade representation of the helicopter dynamics. This differs from many other helicopter models in that it uses an individual blade representation of the rotors, and not a disc. This means that the high-order dynamics of the rotor are captured, instead of the assumption that the rotor tilt is quasi-steady [14].

Although linearising models involve omitting important high order, non-linear dynamics, generally control engineering utilizes linear models of systems, which they base the controller designs upon. It is assumed that a linear model can represent the important rigid body dynamics needed for adequate controller design. This means that a linear model must be formed from the non-linear model described in [13], accomplished using a numerical method found through small perturbation from a given trim condition [14]. This defines a state space system given by:

$$
\begin{aligned}
x & =A x+B u \\
\mathrm{x} & =\left[\begin{array}{lllllllll}
u & v & w & p & q & r & \phi & \theta & \psi
\end{array}\right]^{\mathrm{T}}, \\
\mathrm{u} & =\left[\begin{array}{lllll}
\theta_{0} & \theta_{1 s} & \theta_{1 c} & \theta_{0 t r}
\end{array}\right]^{\mathrm{T}},
\end{aligned}
$$

where, $A$ is the system derivative matrix, $B$ is the control derivative matrix, $u$ (surge), $v$ (sway) and $w$ (heave) are the velocities in the body referenced $\mathrm{x}, \mathrm{y}$, and $\mathrm{z}$ axes respectively, with the rotational velocities $p$ (roll rate), $q$ (pitch rate), and $r$ (yaw rate), and attitudes $\phi$ (roll), $\theta$ (pitch) and $\psi$ (yaw) about those axes. $\theta_{0}$ is the main rotor collective, $\theta_{1 s}$ is the main rotor longitudinal cyclic, $\theta_{1 c}$ is the main rotor lateral cyclic, and $\theta_{0 t r}$ is the tail rotor collective. This model is used for the controller design, but the full representation of the helicopter system is used for testing and evaluating the controllers. For this a PUMA helicopter in a hovering flight is used [15].

\section{Sliding mode control}

SMC is a non-linear control methodology. It has advantages over linear control schemes in that it can be more robust to matched, unmodelled, uncertain system dynamics, and to disturbances [10]. The controllers developed in this paper are of individual decoupled controllers $[16,17]$ that have their to- 
tal control effort comprising of two parts: a linear equivalent term, $u_{\text {equivalent }}$, and a non-linear switching term, $u_{\text {switching: }}$

$$
u=u_{\text {equivalent }}+u_{\text {switching }}
$$

The closed loop system dynamics are represented by a sliding manifold [10]. This sliding manifold is a hyper plane representing zero steady state error, which the controller strives to converge the system toward. The switching term is effective when the system diverges from the zero sliding surface, which causes the system to converge back towards it. The equivalent controller is effective when upon the sliding manifold, representing the desired closed-loop system dynamics.

$$
u_{\text {equivalent }}=-k^{T} x^{\prime} \text {. }
$$

$k$ is the decoupled feedback gain vector found from pole placement [18] and $x$ represents the decoupled system states. The switching term drives the systems when subjected to disturbance or commands, defined by the sliding surface, $\sigma$, which is represented as $[16,17]$

$$
\sigma\left(\Delta x^{\prime}\right)=h^{T} \Delta x^{\prime}=h^{T}\left(x^{\prime}-x_{\mathrm{cmd}}^{\prime}\right),
$$

$x_{\mathrm{cmd}}^{\prime}$ is the desired trajectory. $\sigma$ is a function of the state error, $\Delta x^{\prime}$, and $h$ is the right eigenvector [16] of the desired decoupled closed loop system matrix, $A_{\mathrm{C}}$, found from:

$$
A_{\mathrm{C}}=A^{\prime}-b^{\prime} k^{T} \text {, }
$$

where $A^{\prime}$ is the decoupled system matrix, $b^{\prime}$ is the decoupled input distribution vector. This leads to an appropriate controller function to represent the switching action [16, 17]:

$u_{\text {switching }}=\left(h^{T} b\right)^{-1}\left[h^{T} \dot{x}_{\text {cmd }}^{\prime}-h^{T} \hat{f}(x)-\eta \operatorname{sgn}\left(\sigma\left(\Delta x^{\prime}\right)\right)\right]$,

where, $\hat{f}(x)$ represents the unmodelled dynamics. However, (8) is not very practical, due to system noise and actuator dynamics, resulting in chattering [10]. This is due to small values of $\sigma$ causing the switching term to add a magnitude of $\eta$ to the control action. Consequently this overcompensates for the small error and thus causes the input signal to oscillate around $\sigma=0$. This may result in high actuator wear and may excite any high frequency modes of the system. For this reason, other switching regimes that incorporate a boundary layer $\phi_{\mathrm{BL}}$, around the sliding surface can be used [17]. For this application, a saturation function is employed. This is similar to that of a sgn function in that when $\sigma / \phi_{\mathrm{BL}}>1$, or $\sigma / \phi_{\mathrm{BL}}<-1$, the output of the sat function is the same as the sgn function. However when within the boundary layer, $|\sigma|<\phi_{\mathrm{BL}}$, the output is equal to $\sigma / \phi_{\mathrm{BL}}$. This is known as pseudo (or soft) switching as it removes the hard transition between the sudden transitions from -1 to 1 [17]. This is shown below.

$$
\operatorname{sat}\left(\frac{\sigma}{\phi_{\mathrm{BL}}}\right)=\left\{\begin{array}{ll}
\sigma / \phi_{\mathrm{BL}}>1 & =1 \\
-1>\sigma>1 & =\sigma / \phi_{\mathrm{BL}} \\
\sigma / \phi_{\mathrm{BL}}<-1 & =-1
\end{array} .\right.
$$

When within this region however, there is no guarantee that the sliding surface will be reached [10]. Hence, there must be a trade-off in terms of robustness, performance and chattering. This gives the total control effort as:

$u=-k^{T} x^{\prime}+\left(h^{T} b^{\prime}\right)^{-1}\left[h^{T} \dot{x}_{\mathrm{cmd}}^{\prime}-h^{T} \hat{f}(x)-\eta \operatorname{sat}\left(\frac{\sigma\left(\Delta x^{\prime}\right)}{\phi_{\mathrm{BL}}}\right)\right](10)$

\section{Aeronautical design standards ADS-33}

The ADS-33 document outlines the desired handling qualities of military rotorcraft (see ADS-33 and Cooper Harper for more information [12, 19]). This paper centers upon the design and implementation of an attitude command response type, (offers a lower level of agility than rate response types but tends to offer a higher level of stabilisation). This means that from a step input to the cyclic or pedals, a constant attitude change of pitch, roll or yaw will result, proportional to the magnitude of the step input. The assessment criteria for attitude response types can be broken down into the following areas: small amplitude, moderate amplitude, large amplitude, and inter axis coupling.

As well as the above requirements, the impulse response must be observed. For Attitude hold response types, the attitude should return to trim following a control input. By observing the response following an impulse command, the time for attitude to return to trim is measured. The requirement for pitch, roll and yaw, is that for a pulse controller input, the attitude should return to $10 \%$ of the peak value within 10 seconds. For yaw (heading) there is the additional requirement that for a release of the directional controller the rotorcraft captures the reference heading within $10 \%$ of the yaw rate at release [12],

$$
\psi_{f} \leq \psi_{R} \pm 0.1 r_{R}
$$

or within $1^{\circ}$ of attitude at release:

$$
\psi_{f} \leq \psi_{R} \pm 1^{\circ}
$$

whichever is greater. Here $r_{R}$ is the yaw rate at the time of controller release, $\psi_{R}$ is the yaw attitude at controller release, and $\psi_{f}$ is the final yaw attitude.

Small Amplitude inputs are defined in two parts; Short-term and Mid-term response. The short-term response is defined by bandwidth and phase delay parameters. The bandwidth, $\omega_{\mathrm{BW}}$, is defined to be equal to the phase-limited bandwidth, $\omega_{\text {BWphase }}$ (frequency giving $45^{\circ}$ phase margin) [12]. The phase delay parameter is defined as:

$$
\tau_{p}=\frac{\Delta \Phi 2 \omega_{180}}{57.3\left(2 \omega_{180}\right)}
$$

where $\Delta \Phi 2 \omega_{180}$ is the difference in phase between the $180^{\circ}$ frequency, $\omega_{180}$, and twice the $180^{\circ}$ frequency, $2 \omega_{180}$. However, ADS-33 [12] states that if the gain limited bandwidth, $\omega_{\text {BWgain }}$, is lower than $\omega_{\text {BWphase }}$ (gain margin less than $6 \mathrm{dBs}$ ) then the rotorcraft may be prone to Pilot Induced Oscillation, PIO. This is due to interactions between the helicopter and the pilot that can cause the aircraft to become unstable.

The mid-term response concerns the damping factor, $s$, at frequencies below the bandwidth frequency, $\omega_{\mathrm{BW}}$, found above. It is a measure of the controller's ability to reject unwanted oscillations caused by disturbances, and high order dynamics. ADS-33 states that for Level 1, $s>0.35$ [12].

The moderate amplitude requirements are also known as Attitude Quickness. This is because it is a ratio of peak achievable rate to the peak attitude change. The above ratio can be compared to the ADS-33 criteria for different magnitudes of change in attitude. The criterion is structured so that the over and under shoot characteristics of the attitude response are detrimental. This measure is particularly relevant for rate 
response types where the angular rate of change is controlled. This is because rate systems tend to offer the highest level of agility at the sacrifice of stability. Moderate amplitude requirements are not of major concern because attitude control is a sacrifice of agility to improve stability; hence, attitude quickness is degraded.

Large Amplitude response is important as it helps to assess the craft's ability to retain high levels of handling at points where the non-linearities are most severe [20]. As controllers are typically designed with linear, small-amplitude, approximations of the real system, it is necessary to test the system outside the range where these approximations are valid. The requirements for Aggressive, Target, Acquisition and Track maneuvers (highest specification), for hover and low speed flight are $\pm 30^{\circ}$ for pitch, $\pm 60^{\circ}$ for roll. There is no large amplitude requirement for yaw as the aircraft should be able to perform $360^{\circ}$ rotations indefinitely.

The manner in which pitch is affected by roll, and vice-versa is the inter-axis coupling. For pitch and roll coupling the ratio of roll attitude due to pitch attitude commanded change following a fast input should not exceed \pm 0.25 for Level 1 [12], and vice versa.

\section{Control system}

The control structure employed to implement SMC is shown below.

The system incorporates a model reference block in the form of a low pass pre-filter. This takes the pilot commands, filtering out any high frequency inputs, providing demanded attitudes and rates (6 states). The 3 individual controllers provide the control action. However, as the controllers are decoupled, assuming no cross coupling of the dynamics or actuators, this needs to be taken into account. The unmodelled system dynamics (which include the cross coupling, off axis terms in the system matrix $\boldsymbol{A}$ ), are represented by $\hat{f}(x)$ the term in the controller given in Equation (10). However, for the cross coupling caused by the actuator dynamics (off axis terms in input distribution matrix $\boldsymbol{B}$ ), a pre-compensation matrix is employed. The effect of this is to diagonalise the $\boldsymbol{B}$ matrix by selecting the matrix as follows:

$$
\left[\begin{array}{c}
\theta_{1 c} \\
\theta_{1 s} \\
\theta_{0 t r}
\end{array}\right]=\left[\begin{array}{ccc}
1 & -b_{4,2} / b_{4,3} & -b_{4,4} / b_{4,3} \\
-b_{5,3} / b_{5,2} & 1 & -b_{5,4} / b_{5,2} \\
-b_{6,3} / b_{6,4} & -b_{6,2} / b_{6,4} & 1
\end{array}\right]\left[\begin{array}{c}
\theta_{1 c \mathrm{SMC}} \\
\theta_{1 s \mathrm{SMC}} \\
\theta_{0 t r \mathrm{SMC}}
\end{array}\right],
$$

where $\theta_{\mathrm{SMC}}$ are the respective outputs from the 3 decoupled SMC controllers. The output from the pre-compensator is then added to the trim value that is found in [14] which is valid for the hover flight condition. The controllers themselves are designed as multi-state systems, with each controller design incorporating the appropriate rate and attitude states. It has been found that testing the controller using the state space matrices $\boldsymbol{A}$ and $\boldsymbol{B}$, a very high gain system is realized. However, when testing upon the full helicopter model, this had to be greatly reduced. The high order dynamics of the system become troublesome, and noise, in the form of angular rate transferred from main rotor vibration, necessitates a large reduction of gain, and a large increase in the boundary layer. A hard switching controller is not possible in this system due to this, and therefore a soft switching sat regime is used. Finally the controllers are designed with closed loop poles at 0 and -4 . The input pre-filters have a bandwidth of double the ADS-33 minimum bandwidth [12], with a DC gain of $10 \mathrm{dBs}$. The switching gain and boundary layer are chosen to give stable responses at high amplitude inputs.

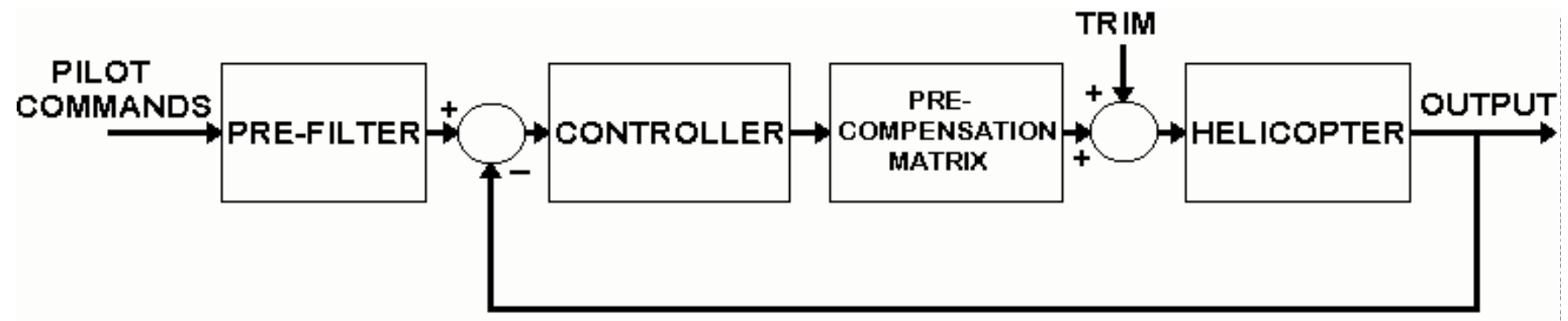

Fig. 1: Control structure diagram
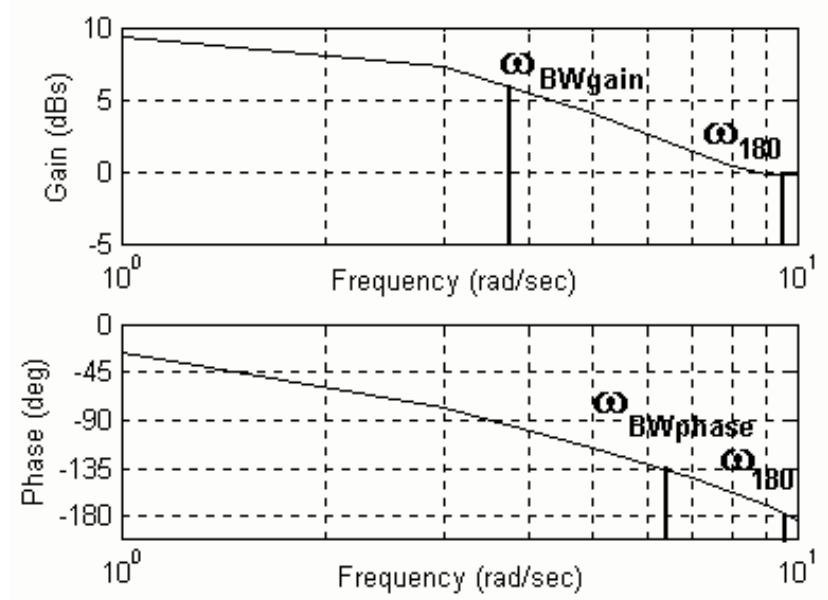

Fig. 2: Pitch Att. Bode plots including $h^{T} \dot{x}_{\text {cmd }}$
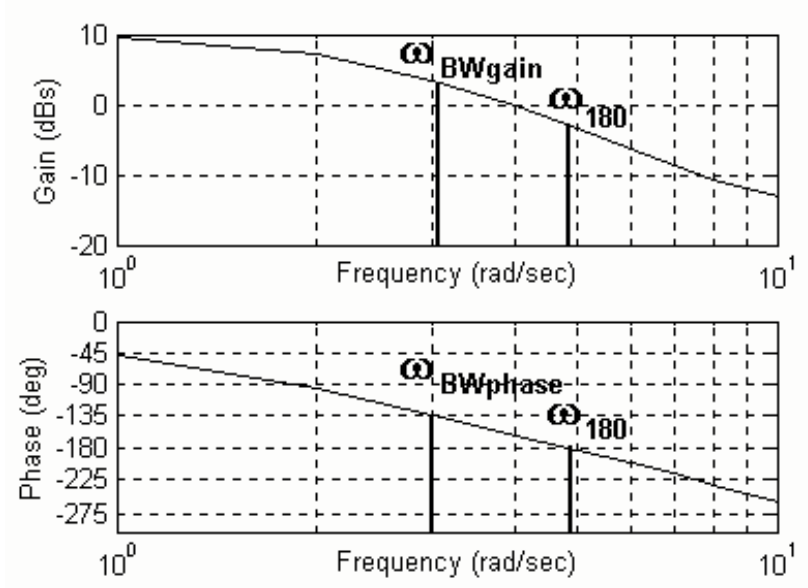

Fig. 3: Pitch Att. Bode plots not including $h^{T} \dot{x}_{\text {cmd }}$ 
During the evaluation of these control schemes, it has been found that the controller produced a system that gives a low gain margin, $\omega_{\text {BWgain }}<\omega_{\text {BWphase }}$, as can be seen in Fig. 2 , which shows bode plots of gain and phase for Pitch between 1 and $10 \mathrm{rad} / \mathrm{sec}$.

This produces a system prone to Pilot Induced Oscillations, as the gain limited bandwidth is lower than the phase limited bandwidth, giving a gain margin of approximately $2.5 \mathrm{dBs}$. This is a highly undesirable attribute for the control system. The way round this problem is to increase the gain roll off characteristic. The most suitable manner to accomplish this is by the removal of the $h^{T} \dot{x}_{\text {cmd }}$ term in the switching function. This term produces a large actuator command from the controller, which gives the system a fast actuation following a command input. The effect of omitting this term is to increase the roll-off of gain and increase the phase delay. This increased phase delay decreases $\omega_{\text {BWphase, which in turn }}$ increases the gain margin, as can be seen in Fig. 3 .

The other effect of this is evident in the moderate amplitude evaluation. When the $h^{T} \dot{x}_{\text {cmd }}$ term is included, Level 1 requirements can be satisfied. However, when the term is omitted, the system does not have the high actuation signal that provides much of the control effort following a command input, resulting in a more sluggish response, as reflected in Figs. 6, 10, and 14 .

Another issue concerning Attitude control is that of steady state tracking. It has been found that the controller given in Equation (10) is insufficient to provide adequate tracking. For this reason it is desirable to include an integral term into the controller.

This results in a new controller given by (15) where $\lambda$ is the integral gain

$u=-k^{T} x^{\prime}+\left(h^{T} b^{\prime}\right)^{-1}\left[-h^{T} \hat{f}(x)-\lambda \int \sigma\left(\Delta x^{\prime}\right)-\eta s a t\left(\frac{\sigma\left(\Delta x^{\prime}\right)}{\phi_{\mathrm{BL}}}\right)\right](15$ )

\section{Pitch control}

The pitch form of the SMC comprises two states, those of pitch rate and pitch attitude. This allows for both rate control and attitude control to be accomplished with the same controller, only requiring different command inputs for each task. The first requirement for an Attitude Hold response type is concerned with the impulse response. Fig. 4 shows the response to a rapid controller impulse. It can be seen that the response is not first order due to the negative overshoot. Also observable is the coupling with roll, which can be seen to be oscillatory, due to the high order rotor dynamics. However, the requirement for returning to trim is met as the pitch returns to $10 \%$ of peak deviation within approximately 2 seconds, and roll and yaw return to $10 \%$ of peak within less than 5 seconds.

The second requirement is that of small amplitude bandwidth and phase delay. Fig. 5 shows the resulting bandwidth and phase delay in relation to the ADS-33 requirements. This indicates that the Level 1 requirement can be satisfied. Bandwidth can be increased by increasing the gain or increasing the bandwidth of the input filter, however, this has the effect of increasing the phase delay due to the on set of high order

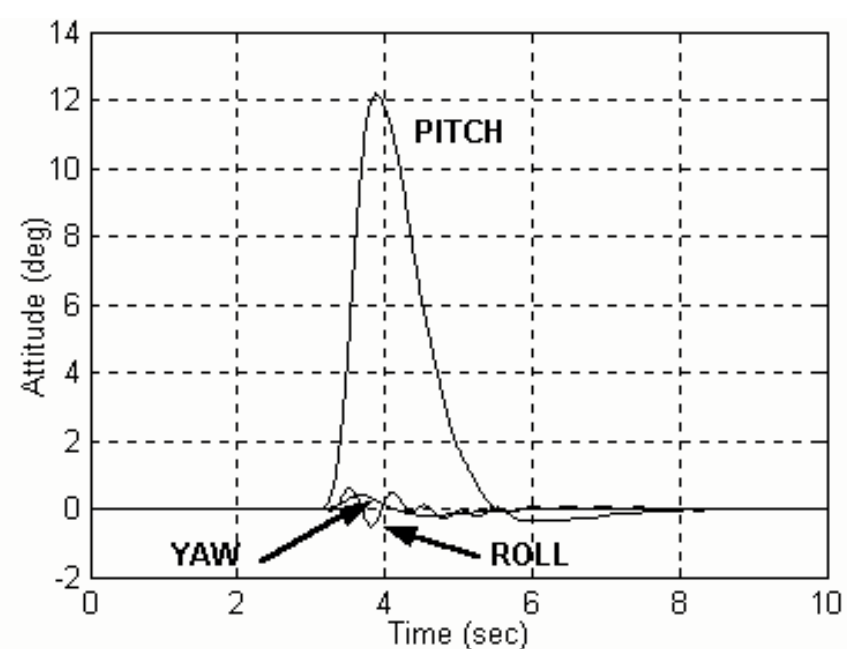

Fig. 4: Attitude response to rapid longitudinal cyclic

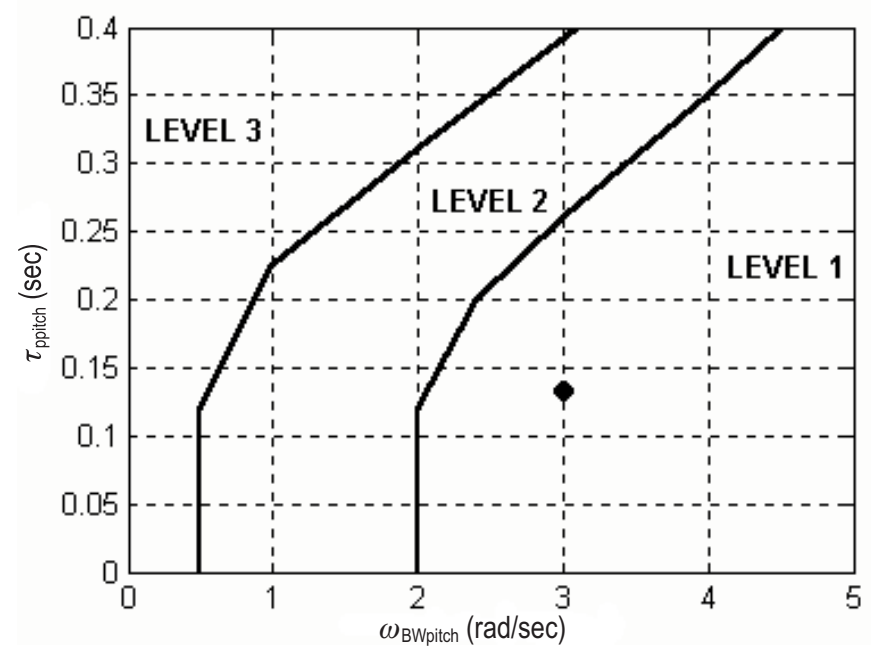

Fig. 5: ADS-33 requirements for pitch attitude [12]

dynamics that are unaccounted for in the controller. These contribute a large decay in phase at high frequencies (over $10 \mathrm{rad} / \mathrm{sec}$ ).

The mid-term requirement is a minimum damping factor of $s=0.35$ in order to satisfy Level 1 handling [1]. The pitch damping is found to be 0.68 , well above the 0.35 requirement. The attainable attitude quickness and the ADS-33 require-

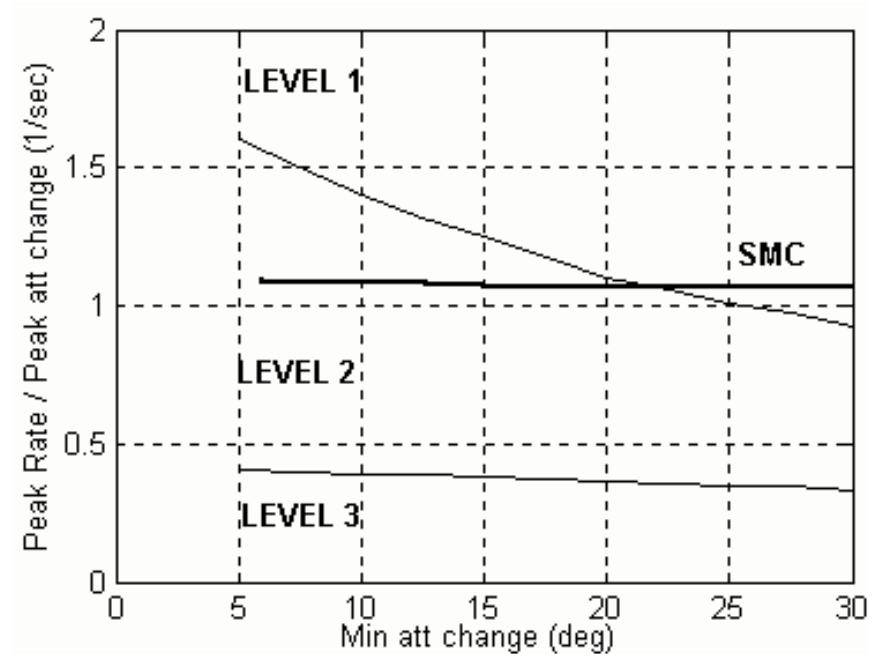

Fig. 6: Moderate amplitude pitch attitude [12] 
ments are shown in Fig. 6. It can be seen that the Level 1 requirements cannot be satisfied over the total range, due to the omission of the $h^{T} \dot{x}_{\text {cmd }}$ term in the controller that provides much of the initial control effort. A constant level in the attitude quickness is not observed in Fig. 6 due to the overshoot seen in at high attitudes (see Fig. 7) which reduces the moderate amplitude response. The large amplitude requirement demands for Attitude control is a minimum of $\pm 30^{\circ}$ [12]. This is shown in Fig. 7 for a positive step in pitch. It should be noted that there is a slight overshoot present in the response, but the coupling between pitch and roll and yaw is

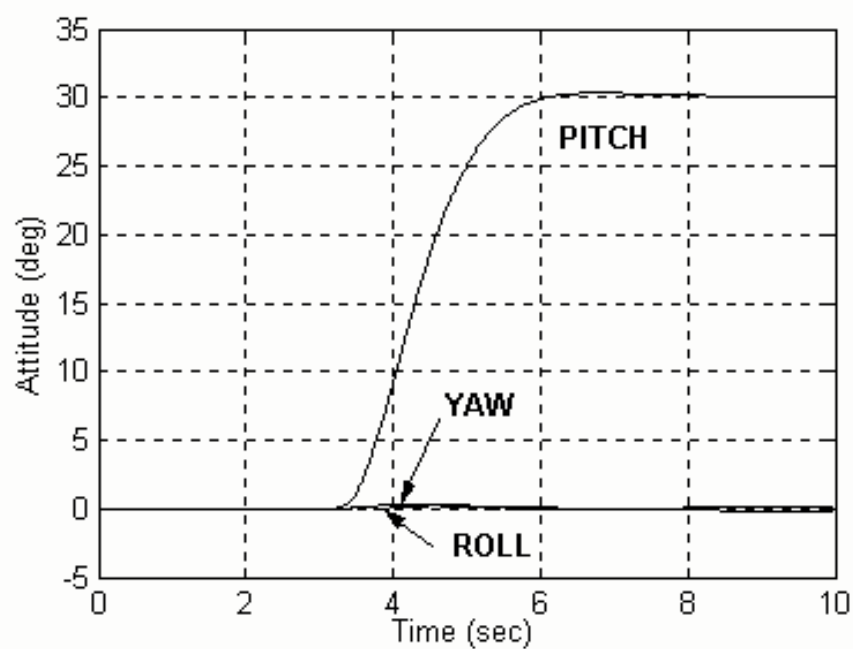

Fig. 7: Large amplitude response of pitch command

minimal.

\section{Roll control}

The first requirement for roll is that of the impulse response. This is shown in Fig. 8. Like that of pitch, the response is not strictly first order due to the negative overshoot. However, it can be seen that this requirement is satisfied as the roll returns to less than $10 \%$ of the peak value in under 3 seconds, far less than the 10 second requirement. Also, as there is little deviation in pitch and yaw, this is not of concern, and demon-

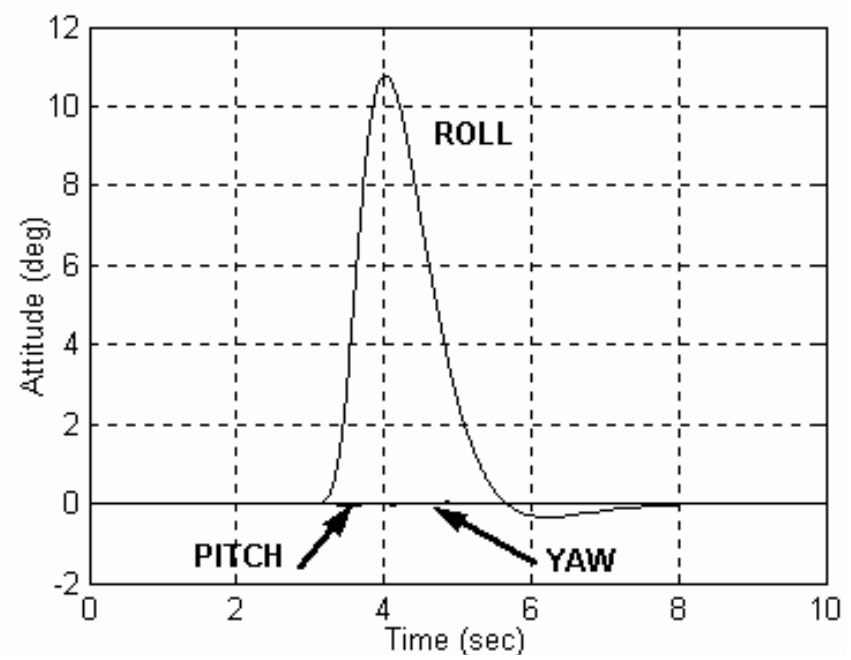

Fig. 8: Attitude response to rapid lateral cyclic input

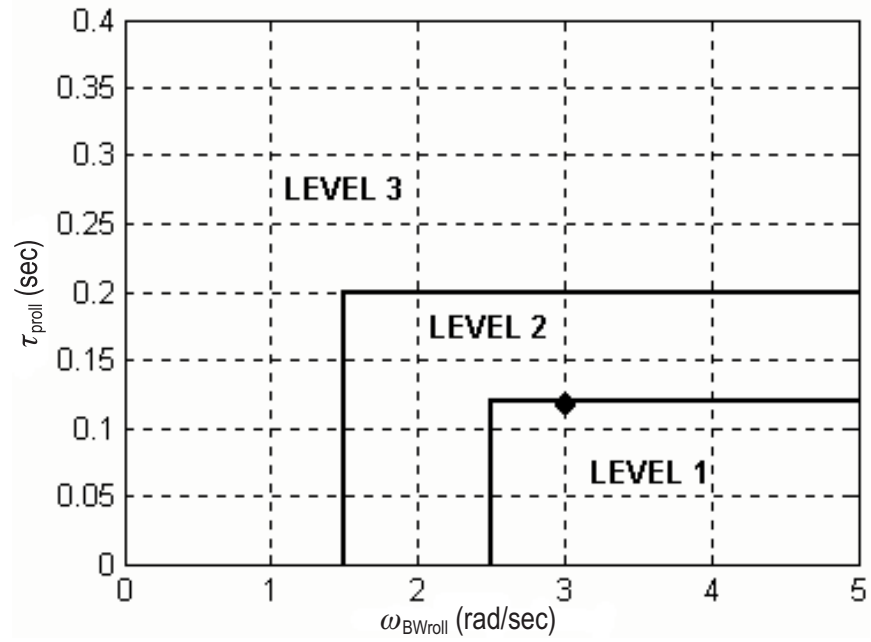

Fig. 9: ADS-33 requirements for roll attitude [12]

strates the successful decoupling of pitch and yaw from the roll channel.

The phase delay in roll is the most stringent, with a required phase delay of under 0.12 sec for Level 1. Fig. 9 shows the resulting bandwidth and phase delay in relation to the ADS-33 requirements, and that the Level 1 requirement is met, although further tuning is required to reduce the phase delay $\tau_{\mathrm{p}}$.

For mid-term response, the roll damping is found to be 0.70 . The moderate amplitude requirements in roll are the most stringent. The attainable attitude quickness and the ADS-33 requirements are shown in Fig. 10. It can be seen that the Level 1 requirements cannot be satisfied over the total whole range, due to the omission of the $h^{T} \dot{x}_{\text {cmd }}$ term in the controller that provides much of the initial control effort. A constant level in the attitude quickness is not observed in Fig. 10. The level of overshoot observed in the roll response does not increase greatly as a function of commanded input (overshoot only varies from $1^{\circ}$ to $2^{\circ}$ over the $10^{\circ}$ to $60^{\circ}$ attitude range) due to the effect of the integral action improving the steady state tracking. This means that the ratio of peak rate to maximum attitude change is lower for small attitude changes than for large attitude changes. Consequently, the moderate amplitude response is poorer in the lower half of the attitude

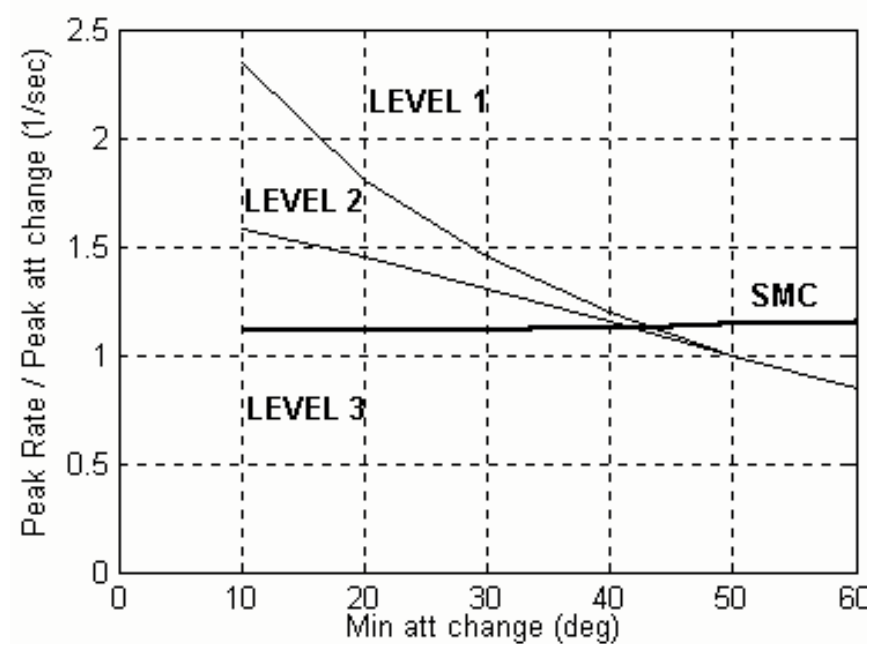

Fig. 10: Moderate amplitude of roll attitude [12] 


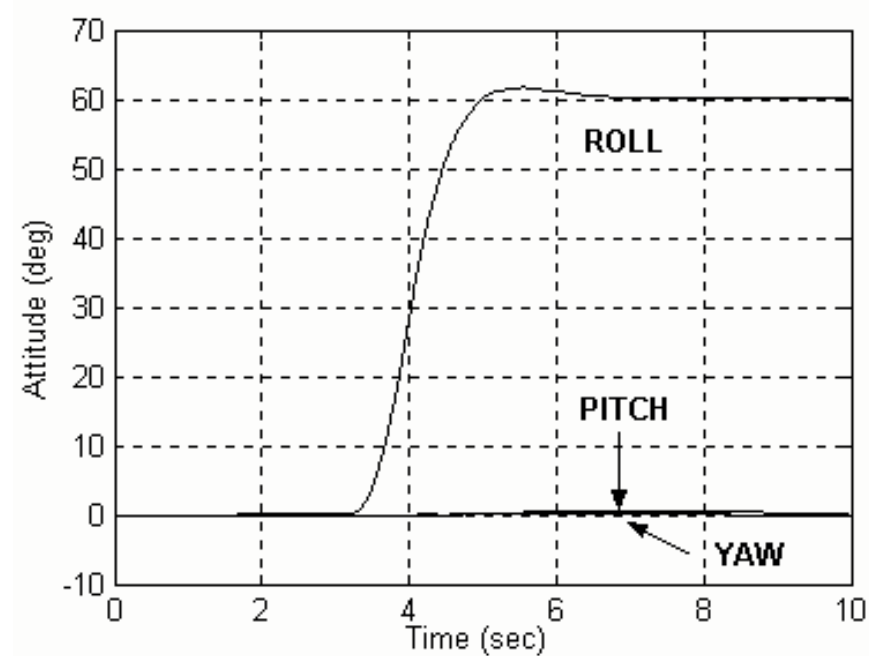

Fig. 11: Large amplitude response of roll

range. The large amplitude requirement demands are for Rate control, a minimum of $\pm 50 \%$ sec [12]. Fig. 11 shows a positive step command in roll. It should be noted that there is overshoot present in the response, some high frequency oscillation, and the coupling between roll, pitch and yaw. As with pitch, this coupling can be improved upon by increasing the gain of the off-axis controllers at the expense of the commanded responses in those axes.

\section{Coupling}

Interaxis Coupling is defined as a ratio of roll due to pitch, and vice-versa. For coupling of pitch due to roll, a ratio of 0.008 is attained, and for roll due to pitch, a ratio of 0.022 is found. This is well below the 0.25 Level 1 requirement [12].

\section{Yaw control}

Yaw attitude (or heading) control has essentially the same requirements applied to it as pitch and roll at hover and low speeds (under $40 \mathrm{kts}$ ). The first requirement, like that of pitch and roll, is that the yaw attitude should return to trim within 10 seconds of a rapid controller input. As can be seen from

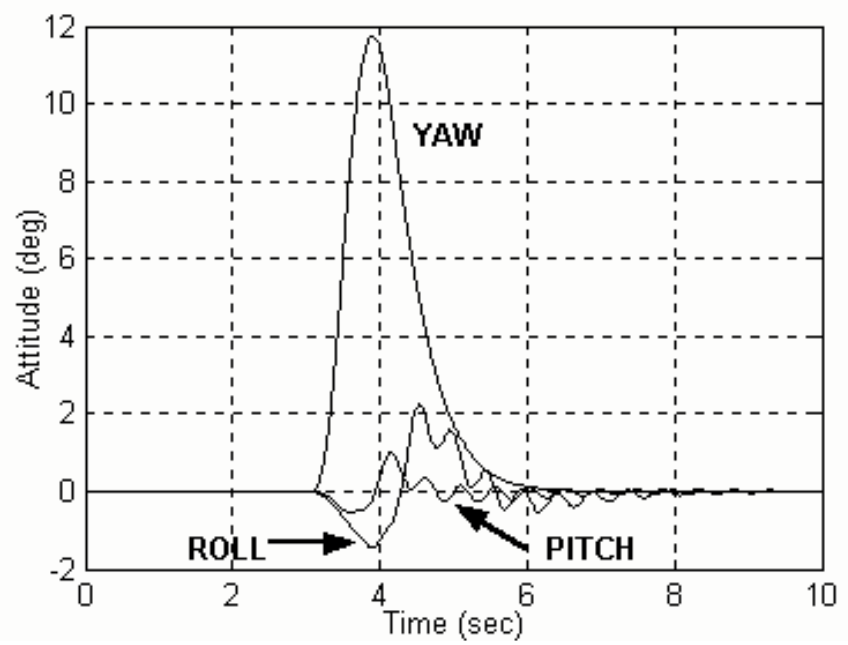

Fig. 12: Attitude response to rapid tail rotor cyclic input
Fig. 12, this requirement can be met. Yaw control exhibits superior damping to that of pitch and roll as there is minimal overshoot, and the response approximates a first order be-

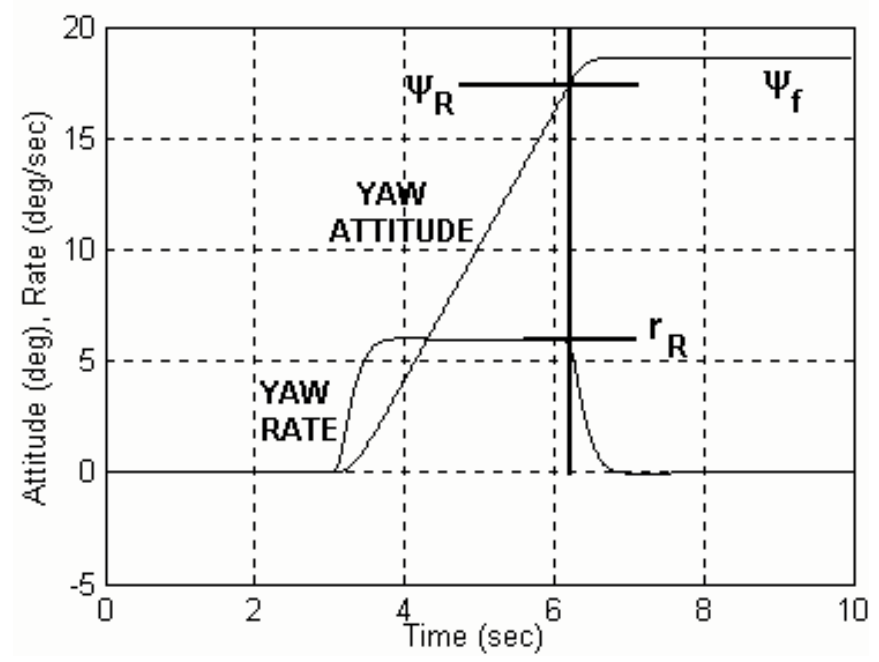

Fig. 13: Yaw attitude (heading) capture

haviour. However, it can be seen that coupling of pitch and roll from this axis is still present, due to the deviation of both attitudes from trim following the input.

The additional requirement for yaw attitude hold is that of capturing the reference heading within $10 \%$ of the yaw rate following the release of a commanded yaw input. Fig. 13 shows the response to a command resulting in a step change in yaw rate, which is rapidly applied and released. The final yaw attitude, $\psi_{\text {, }}$ should remain within the greater of $\psi_{R} \pm 1^{\circ}$ or $\psi_{R} \pm 0.1 r_{R}$. It is found that for the $6^{\circ} \mathrm{sec}$ yaw rate, and a release attitude of $18^{\circ}, \psi_{f}$ is $18.5^{\circ}$, which is within the $10 \%$ of yaw rate at time of release $(0.6 \mathrm{deg} / \mathrm{sec})$ and well under the $1^{\circ}$ requirement.

The yaw bandwidths requirements are the highest, with a required bandwidth of at least $3.5 \mathrm{rad} / \mathrm{sec}$. Fig. 14 shows the resulting bandwidth and phase delay in relation to the ADS-33 requirements. This shows that the Level 1 requirement is satisfied.

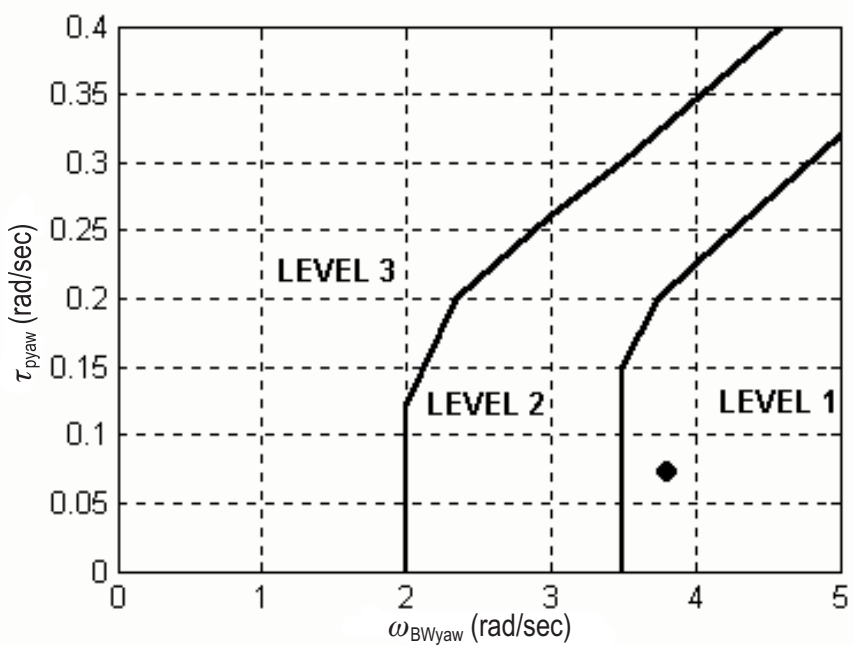

Fig. 14: ADS-33 Bandwidth for Yaw Attitude [12] 


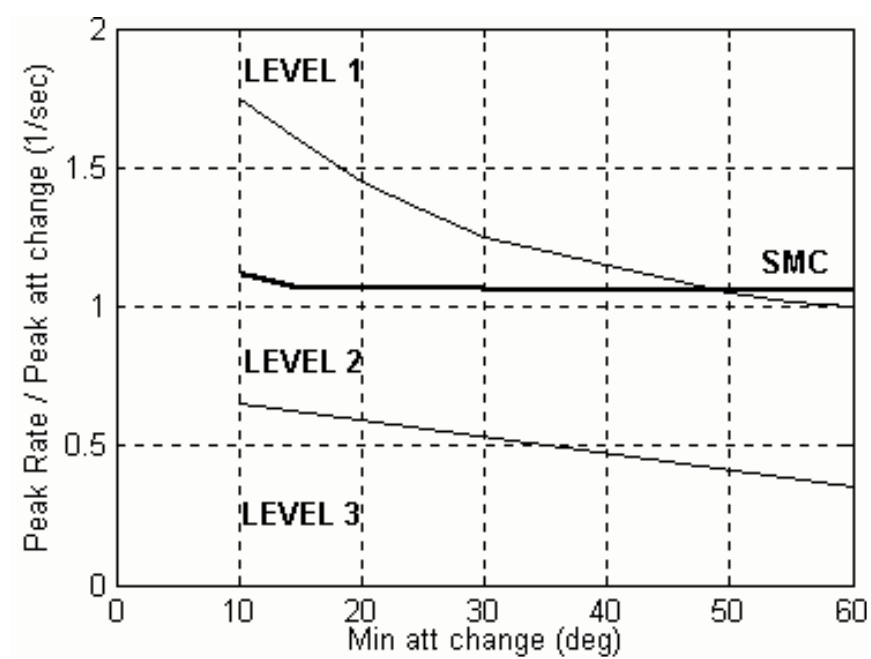

Fig. 15: Moderate amplitude of yaw attitude [12]

For mid-term response, the yaw damping has been found to be 0.9 . The attainable attitude quickness and the ADS-33 requirements are shown in Figure 15. It can be seen that the Level 1 requirements cannot be satisfied, due to the omission of the $h^{T} \dot{x}_{\text {cmd }}$ term in the controller that provides much of the initial control effort. It must be noted here that these results are for negative yaw commands. Although the resulting attitude quickness is very similar for positive steps, at inputs over $40^{\circ} / \mathrm{sec}$ the system can become unstable. This is likely to be due to the asymmetry of the aircraft from the direction of rotation of the main rotor, placement of the tail rotor, and the effect of wake caused by the former on the latter, which excites coupling between the yaw axis and roll axis.

\section{Conclusions}

In this paper, the successful implementation of a sliding mode attitude control system has been presented. The assessment of these controllers to the ADS-33 requirements has shown that Level 1 handling can be achieved for pitch, roll and yaw, creating a high bandwidth, high gain, stable control platform. Despite the use of a decoupled control strategy, the coupling between the channels is well within acceptable levels. Although the controller design does not take into account the high order dynamics, the testing of the controllers shows that the SMC system can cope with such dynamics. However, there is room for improvement in this system. Many of the high order dynamics can be observed on the responses, and removal of these would be desirable. The issue of attaining sufficient gain margins to avoid PIO has been addressed by altering the controller structure, but at the cost of increasing phase lag, reducing gain, and reducing available bandwidth, resulting in a less agile system as reflected in the moderate amplitude measurement. The systems exhibit high levels of damping and allow for large amplitude while maintaining stable control and low levels of coupling.

\section{References}

[1] Manness, M. A., Gribble, J. J., Murray-Smith, D. J.: "Helicopter Flight Control Law Design Methodologies", September 1991.
[2] Prouty, R. W.: "Helicopter Control Systems: A History", Journal of Guidance, Control, and Dynamics, Vol. 26 (2003), No. 1, January-February 2003.

[3] Ingle, S. J., Celi, R.: "Effects of Higher Order Dynamics on Helicopter Flight Control Law Design", presented at the Annual Forum of the American Helicopter Society, Washington, D. C., June 1992.

[4] Manness, M. A., Murray-Smith, D. J.: “Aspects of Multivariable Flight Control Law Design for Helicopters Using Eigenstructure Assignment." Journal of the American Helicopter Society, Vol. 37 (1992), No. 3, July 1992.

[5] Tischler, M. B.: "Digital Control of Highly Augmented Combat Rotorcraft." NASA-Technical Memorandum 88346, USAAVSCOM Technical report 87A-5 May 1987.

[6] Bag, S. K., Spurgeon, S. K., Edwards, C.: "Robust Sliding Mode Design Based upon output Feedback." Conference Publication No. 427, UKACC International Conference on Control, 2-5 September 1996, p. 406-411.

[7] Fossard, A. J.: "Helicopter Control Law Based on Sliding Mode with Model Following." International Journal of Control, Vol. 57 (1993), No. 5, p. 1221-1235.

[8] Sira-Ramirez, H., Zribi, M., Ahmad, S.: "Dynamical Sliding Mode Control Approach for Vertical Flight Regulation in Helicopters.” IEE Proc.-Control Theory Appl., Vol. 141, No. 1, January 1994, p. 19-24.

[9] Spurgeon, S. K., Edwards, C., Foster, N. P.: "Robust Model Reference Control Using a Sliding Mode Controller/Observer Scheme with Application to a Helicopter Problem." IEEE Workshop on Variable Structure Systems, 1996.

[10] Edwards, C., Spurgeon, S. K.: Sliding Mode Control: Theory and Applications. Taylor and Francis Ltd, London 1998.

[11] Slotine, J. J. E., Li, W.: Applied Non-linear Control. Prentice Hall, 1992.

[12] ADS-33E-PRF, “Aeronautical Design Standard, 33E, Handling Qualities Requirements for Military Rotorcraft", United States Army Aviation and Missile Command, Aviation Engineering Directorate, Redstone Arsenal, Alabama, 21 ${ }^{\text {st }}$ March 2000.

[13] Houston, S. S.: Rotorcraft Aerodynamics Simulation for Control Analysis - Mathematical Model Definition. University of Glasgow, Dept. of Aerospace Engineering Internal Report No. 9123, 1991.

[14] Houston, S. S.: "Validation of a Non-Linear Individual Blade Flight Dynamics Model Using a Perturbation Method." The Aeronautical Journal of the Royal Aeronautical Society, Vol. 98 (1994), No. 977, August-September 1994.

[15] Houston, S. S.: "Validation of a Blade-Element Helicopter Model for Large Amplitude Manoeuvres." The Aeronautical Journal of the Royal Aeronautical Society, Vol. 101 (1997), No. 1001, January 1997, p. 1-7.

[16] Healey, A. J., Lienard, D.: "Multivariable Sliding Mode Control for Autonomous Diving and Steering of Unmanned underwater Vehicles." IEEE Journal of Oceanic Engineering, Vol 18 (1993), No. 3, p. 327-339. 
[17] McGookin, E. W.: "Sliding Mode Control of a Submarine.” M. Eng thesis, University of Glasgow, Dept. E\&EE Engineering, 1993.

[18] Kautsky, J., Nichols, N. K., Van Dooren, P.: "Robust Poles Assignment in Linear State Feedback." International Journal of Control, Vol. 41 (1985), No. 5, p. 1129-1155.

[19] Cooper, G. E., Harper Jr, R. P.: "The Use of Pilot Rating in the Evaluation of Aircraft Handling Qualities." NASA-TN-D-5153, April 1969.

[20] Osder, S., Caldwell, D.: "Design and Robustness Issues for Highly Augmented Helicopter Controls." Journal of Guidance, Control and Dynamics, Vol. 15 (1992), No. 6, November-December 1992.
David J. McGeoch

phone:+441413306137

fax: +44 1313306004

email: d.mcgeoch@elec.gla.ac.uk

\section{Dr. Euan W. McGookin}

Center for Systems and Control

Department of Electronic and Electrical Engineering

University of Glasgow, Glasgow, G12 8LT, UK

Dr. Stewart S. Houston

Rotorcraft Flight Dynamics Group

Department of Aerospace Engineering

University of Glasgow, G12 8QQ, UK 